\title{
EL FUTURO ACADÉMICO DEL PERIODISMO MEXICANO: LA UAM
}

\section{THE ACADEMIC FUTURE OF JOURNALISM MEXICAM: THE U.A.M.}

David Caldevilla Domínguez ${ }^{1}$. Universidad Complutense de Madrid. España. davidcaldevilla@ccinf.ucm.es

Enrique García García. Radio Intereconomía. España. sleatertr@hotmail.com

\section{RESUMEN}

Este artículo tiene fines generales respecto al establecimiento del estado de la cuestión académica acerca de la carrera Ciencias de la Comunicación y Periodismo en el ámbito de las licenciaturas nacionales en México, clasificadas en función de la demanda de matriculaciones, y su resultado a la hora de aplicarse al mercado de trabajo. Con posterioridad, se trata la carrera en el contexto de las 101 ofertas académicas de la UNAM y el perfil de sus alumnos (diferenciados entre solicitantes y admitidos), y cómo esta continua siendo una de las rutas de estudio más demandas.

PALABRAS CLAVE: Ciencias de la Comunicación y Periodismo, demanda, matrícula, mercado laboral, perfil del estudiante.

\section{ABSTRACT}

This article has generic goals towards setting the academic matter's situation about the "Communication sciences and journalism" degree in the sphere the national mexican degrees, classified according to their demand and results in the labour market. Afterwards the degree is observed in the context of the 101 academic offers in the UNAM and their alumni profiles (both soliciters \& admitted) and how this keeps being one of the most demanded degrees.

KEY WORDS: Communication Sciences and Journalism, demand, registration, labor market, student profile.

\section{Cómo citar el artículo:}

Caldevilla Domínguez, D. y García García E. (2015). El futuro académico del periodismo mexicano: la UAM. Revista de Ciencias de la Comunicación e Información, 20, 1-7.

DOI: http://doi.org/10.35742/revistacccomunicacioneinformacion.2015.20.1-7

\footnotetext{
${ }^{1}$ David Caldevilla Domínguez: Universidad Complutense de Madrid. Departamento CAP 2. España.
} Recibido: 01/03/2015 - Aceptado: 16/04/2015 - Publicado: 15/12/2015 


\section{Periodismo y ciencias de la información a nivel nacional}

Nos referimos a una carrera clasificada la número trece en cantidad de matriculaciones (DGPP, 2012) contabilizando cifras de las 30 principales entidades universitarias de México (ANUES, s/f). De acuerdo con la Secretaría de Educación Pública, en el curso 2011-2012 (DGPP) de entre casi tres millones de alumnos totales, se matricularon en periodismo y CC de la comunicación más de 65000 .

En decimoquinta posición con respecto a la citada clasificación, es importante señalar que se encuentra "Técnicas audiovisuales y producción de medios" una carrera con una innegable vinculación a nuestro campo de estudio. Tener en cuenta esas cifras elevaría el interés por el sector periodístico entre el alumnado mexicano hasta 129.000 matriculados: entre las dos, ambas carreras podrían ocupar el tercer puesto en número de ingresos.

La consecuencia social más directa y visible de estas sencillas cifras, se ve al compararlas con el progresivo y consabido deterioro del mercado laboral: el elevado número de licenciados se ve obligado a luchar por unos puestos de trabajo decrecientes tanto en número como en calidad.

Esta sobre-oferta de profesionales (o infra-oferta de trabajo) desemboca en numerosos casos de licenciados resignándose a aceptar empleos alejados de su duramente ganada formación. Según la Encuesta Nacional de Ocupación y Empleo de México, el 9\% de los encuestados ocupan puestos técnicos por debajo de su cualificación superior. Y un $57 \%$ de los estudiantes de periodismo directamente no trabaja en nada relacionado con su carrera (Encuesta Nacional de Ocupación y Empleo, STPS-INEGI, cifras trimestrales). Con todo, en México, la carrera es la décima con más licenciados empleados a nivel nacional, aunque el $60 \%$ de ello no lo estén en tareas relacionadas con sus estudios. Por estos mismos datos, el número de periodistas con trabajo (editorial o no) aumentó un $28 \%$ en el periodo de 2005 a 2012.

El Observatorio Laboral define a esta carrera con las siguientes palabras: "Los estudios que comprenden los aspectos científicos, humanísticos y críticos de la comunicación humana y colectiva en una variedad de formatos, medios y contextos." Considera también los programas que se centran en la teoría y la práctica de la recolección, procesamiento y distribución de noticias y reportajes de interés públicos (2013).

Esta definición resulta imprecisa y genérica, siendo el germen de una innecesaria complicación: y es que existen múltiples nomenclaturas (del orden de 25) para este grado universitario: denominaciones que muy a menudo van acompañadas de planes de estudio significativamente diferentes en su composición, intensidad y enfoque. Así, por ejemplo, la carrera puede encontrarse bajo los epígrafes de:

- Comunicación e información

- Comunicación organizacional

- Ciencias de la comunicación

- Comunicación

- Comunicación y periodismo

- Comunicación y arte digital 
- Periodismo

- Comunicación y medios digitales

- Ciencias de la información

- Comunicación social

- Comunicación humana

- Periodismo y medios de información

- Arte digital

- Ciencias y técnicas de la comunicación

- Comunicación y artes audiovisuales

- Periodismo y comunicación públicas

- Animación, arte digital y multimedia

- Comunicación audio visual

- Comunicación periodística

- Comunicación, publicidad y relaciones públicas

- Comunicación y tecnología educativa

- Comunicación y medios

- Ciencias de la información y la comunicación

- Multimedia y animación digital

- Comunicación visual

- Comunicación e imagen pública

- Comunicación y producto de medios

- Comunicación e innovación educativa (ANUIES, 2012)

A parte de estos, hay otra serie de cifras que es importante anotar para tener una visión de conjunto: de entre todos los matriculados del curso 2011-2012, casi el $60 \%$ eran mujeres, y el 40,6\% varones. La cantidad de graduados durante ese tiempo ascendió a 12254, siendo la decimosegunda carrera en número de licenciados (SEP, s/f).

Los ingresos promedio de los estudiantes (Encuesta Nacional de Ocupación y Empleo, STPS-INEGI s/f) son comparativamente bajos: los cuadragésimos segundos en el ranking nacional.

La carrera se cursa en más de 30 universidades y centros superiores. En DF solamente, en:

- Instituto Tecnológico y de Estudios Superiores de Monterrey

- Universidad de las Américas, A.C.

- Universidad Iberoamericana

- Universidad Intercontinental

- Universidad La Salle, A.C.

- Universidad Panamericana

- Universidad Autónoma Metropolitana

- Universidad Nacional Autónoma de México

\section{La UNAM: Periodismo y Ciencias de la Información}

La Universidad Nacional Autónoma de México, UNAM, es la Institución de Educación Superior, IES, más importante del país, por su trayectoria histórica, 
científica, académica y cualitativa. En el curso 2012-2013, tiene casi 200.000 alumnos (DGAE UNAM 2013) y oferta 101 licenciaturas.

Y siguiendo la tendencia antes establecida, en ella el periodismo se encuentra entre las 15 carreras más demandadas, aunque arrastra un lento declive de cinco años, lo que nos dirige indirectamente hacia la influencia de las universidades sobre las preferencias de los estudiantes (Osorio, 2013) pero también y de una manera bien distinta junto con los datos de desempleo, a las reflexiones en torno al impacto de las TIC en la universidad realizadas por Isabel González Flores y Marlery Sánchez Díaz (2012).

En febrero de 2013 , el $29 \%$ de los que se matricularon en ella optaron por las Ciencias sociales: Contabilidad, gestión, Relaciones internacionales, derecho, comunicación y periodismo. Por Biología y ciencias de la salud se decantó el $37,63 \%$. Física y matemáticas e ingenierías, un $20,88 \%$ y arte y humanidades un 11\% (DGAE, UNAM, 2013).

Podemos coligar de estos datos que tanto Periodismo como todas las titulaciones relacionadas con la comunicación tienen una alta demanda, encuadrada dentro de la que a su vez tienen las Ciencias Sociales. Todo ello a costa de Física, matemáticas, o las vitales ingenierías. En estas escasean los profesionales autóctonos, provocando una dependencia de los existentes y de los externos.

Según la SEP (2012) las candidaturas en matemáticas durante el ciclo 2011-2012 ascendieron a 7683, y algo más de 6000 en física. Para Ciencias de la Comunicación, la UNAM tiene tres planes de estudio distintos (DGOSE, 2012) lo que enlaza con la tendencia a la especialización de los medios y profesionales (De Haro, 2011):

1. Facultad de Ciencias Políticas y Sociales, (Licenciado en Ciencias de la Comunicación) modalidad escolarizado y Sistema de Universidad Abierta y Educación a Distancia. Dura 9 semestres, con especialidades en: Producción Audiovisual, Publicidad, Comunicación Política, Periodismo en los Medios y Comunicación Organizacional.

2. Facultad de Estudios Superiores Acatlán, (Licenciado en Comunicación) Sistema Escolarizado. 9 semestres, con las especialidades de: Comunicación Organizacional, Investigación y Docencia, Medios Electrónicos y Periodismo Escrito.

3. Facultad de Estudios Superiores Aragón, (Licenciado en Comunicación y Periodismo.) Sistema Escolarizado. 8 semestres: especialidades: Trabajo Periodístico Escrito, Producción Radiofónica y Producción Televisiva.

\section{Los estudiantes}

En base a una selección de las variables e indicadores aportados por la Dirección General de Planeación (DGPL) y publicados en el Portal de Estadística Universitaria (UNAM, 2008) podemos dar una pauta del perfil de los estudiantes que optan por esta carrera en la UNAM.

Las variables mencionadas son: Carrera y Plantel, Ingreso por Pase reglamentado y por examen de selección; Datos generales: edad, sexo, estado civil; Datos estudios 
anteriores: escuela o plantel de procedencia, estudios terminados en 3 años, promedio de calificaciones, exámenes extraordinarios; Datos personales: insistencia de los padres en los estudios; Datos situación socieconómica: nivel de estudios de la madre y del padre, principal ocupación de la madre y del padre; principal sostén económico, ingreso familiar mensual; Datos de bienes y servicios: computadora personal, automóvil, teléfono celular, televisión de paga; y Datos de la situación laboral del estudiante.

En el curso 2008-2009, el periodismo en la UNAM se contaba entre las diez carreras con más solicitudes de ingreso (la séptima con más solicitudes): 7400 estudiantes del total de los 172.000 alumnos. Un 4\% del total. (DGAE UNAM, 2010).

El número de alumnos que entró en la carrera en el Sistema Escolarizado, durante dicho curso, fue de 1.527: el 22,3\% del total de estudiantes de la misma carrera que fue de 6.819 (DGAE, UNAM, 2010).

Por otra parte, de acuerdo al Perfil de aspirantes y aceptados a la licenciatura Ciencias de la Comunicación en el 2008, (Coordinación de Planeación, Dirección General de Planeación) de 1381 alumnos encuestados, el 45,03\% ingresó a la Facultad de Ciencias Políticas y Sociales, 26,14\% a la Facultad de Estudios Superiores Acatlán y $28,81 \%$ a la Facultad de Estudios Superiores Aragón. De ese total, el $74 \%$ accedió mediante pase reglamentado y el $25 \%$ por examen. La edad media del alumnado iba de los 18 a los 21 años (88,7\%) el 11,3\% restante se movía entre las siguientes edades: 17, de 22 a 25 y más de 26 años. Solteros $97,9 \%$, y $2,1 \%$ casados o en estado civil similar con una proporción más alta de mujeres $60,3 \%$.

La frecuencia relativa a la escuela de origen de los alumnos que accedieron por pase reglamentado no es significativa, pues provienen de todos los planteles del bachillerato UNAM, sin embargo, podemos apuntar que el 34,7\% de los alumnos que accedieron a esta carrera provienen de la Preparatoria № 5 y los Colegios de Ciencias y Humanidades Naucalpan, Oriente y Vallejo.

En relación a la escuela de procedencia de los que ingresaron por examen de selección el $65,1 \%$ estudiaron el bachillerato público, 3,7\% privado y $30,5 \%$ en ambos. Terminaron en tres años el bachillerato el $75,8 \%$ de todos los que accedieron a la carrera, y el $21,2 \%$ restante no lo hizo, por motivos económicos, por problemas emocionales, por causas académicas, etc. La nota media fue de 7,5 a 8,5 $(55,7 \%)$; el $57,7 \%$ no se tuvo que presentar a ningún examen extraordinario; el 80,3\% consideró importante para sus estudios la insistencia paterna; el nivel máximo de estudios de la madres de los alumnos es de licenciatura o Normal Superior un $26,8 \%$ de las veces y carrera técnica un $21,7 \%$; ara los padres: licenciatura o Normal Superior corresponde a un $34,6 \%$ y bachillerato o similar $25,0 \%$; la principal ocupación de las madres es empleada por cuenta ajena $(30,6 \%)$ y ninguno $(31,2 \%)$; la principal ocupación del padre es empleado por cuenta ajena $(37,7 \%)$ tendero $(19,3 \%)$ y autónomo (15,2\%).

Los ingresos familiares mensuales medios están entre 3.447.60 \$ y 10.342.80\$, para el $55 \%$. 


\section{Conclusiones}

El desequilibrio laboral de México llega hasta la formación superior, y a los centros universitarios privados y públicos. Lo hemos visto con la Universidad Nacional Autónoma de México, en la que el gran abanico de opciones académicas no mitiga esta tendencia al desempleo final.

Ciencias de la Comunicación y Periodismo es elegida como opción de estudios sobre todo por el alumnado femenino; y cuenta con múltiples vías de abordaje académico en cuanto a universidades y programas específicos de estudio.

Como el $60 \%$ de los licenciados en este grado terminan llevando a cabo labores diferentes a los previstos en su formación, está claro que existe una relación no exclusiva entre la alta demanda académica de la carrera y la crónica escasez de puestos laborales asociados con, además, bajas remuneraciones.

Según los datos vistos sobre la UNAM, sabemos que de entre los estudiantes que se postulan a ella, casi la mitad busca entrar en la Facultad de Ciencias Políticas y Sociales; el $75 \%$ lo hace por pase reglamentado, y la mayoría son mujeres de edades comprendidas entre los 18 y 21 años. Buena parte de ellas proceden del Bachillerato público, con una nota media de notable.

\section{Referencias}

ANUIES, (s/f). En "Oferta Educativa 2012". Observatorio Laboral. Recuperado de www.observatoriolaboral.gob.mx.

DE HARO-DE SAN MATEO, Ma ${ }^{a}$ V. (2011). La evolución estratégica de la prensa especializada en la historia del periodismo. Un estudio de caso, en Revista de Comunicación de la SEECl, XV(25). Recuperado de www.seeci.net/revista/index.php/seeci/article/view/130

DGAE (1) UNAM (2013). Serie Estadística UNAM 2000-2013. En: Portal de Estadística Universitaria, UNAM. Recuperado de www.estadística.unam.mx/series inst/index.php

DGAE (2) UNAM (2013). Oferta de lugares por carrera y plantel, para el concurso de selección de febrero de 2013. Recuperado de https://servicios.dgae.unam.mx/febrero2013/ofertalugares/oferta febrero2013.ht $\underline{\mathrm{ml}}$

DGOSE UNAM, (2012). Guía de Carreras UNAM 2012-2013. Ed. DGOSE, UNAM. Recuperado de www.escolar.unam.mx/pdfs/formasdeingreso13web.pdf

DGPP (2012). Sistema de Consulta y explotación. Educación superior. En "Matrícula escolarizada en programas de licenciatura agrupadas por área", Dirección General de Educación Superior Universitaria, SGESU-SEP. Recuperado de www. dgesu.ses.sep.gob.mx 
GONZÁLEZ-FLORES, I. (2012). Necesidad de la alfabetización informacional en la educación superior, en Revista de Comunicación Vivat Academia, Año XV, 121. Recuperado de www.vivatacademia.net/index.php/vivat/article/view/19

OBSERVATORIO LABORAL (2013). Comunicación y periodismo. Recuperado de www.observatoriolaboral.gob.mx

OSORIO, J. L. (2013). La comunicación en el empoderamiento de la cultura emprendedora, en Revista de Comunicación de la SEECI, XVII(30). Recuperado de www.seeci.net/revista/index.php/seeci/article/view/6/pdf 20

SÁNCHEZ-DÍAZ, M. (2012). La gestión de competencias informacionales en las universidades: reto para los profesionales de la información, en Revista de Comunicación Vivat Academia, Año XV, 121. Recuperado de www.vivatacademia.net/index.php/vivat/article/view/18

SEP (1), (s/f). En Comunicación y periodismo. Matrícula y Egreso (Ciclo Escolar 2011-2012). Observatorio Laboral. Recuperado de www.observatoriolaboral.gob.mx

SEP (2), (s/f). En las carreras de: Matemáticas y Física. "Matrícula y Egreso" (ciclo escolar 2011-2012). Observatorio Laboral. Recuperado de www.observatoriolaboral.gob.mx

STPS-INEGI, Encuesta Nacional de Ocupación y Empleo (cifras trimestrales) (2013). En "Indicadores", Observatorio Laboral. Última modificación: Abril 2013. Recuperado de www.observatoriolaboral.gob.mx

UNAM (2008). Portal de Estadística Universitaria. Perfiles alumnos de primer ingreso a la UNAM. Recuperado de www.estadistica.unam.mx/perfiles/ 